\title{
Témoignages du Strasbourgeois Victor Stoeber sur la médecine parisienne (1824-1825)
}

\author{
Par Th. Vetter
}

Il était d'usage, naguère, chez les Strasbourgeois qui voulaient acquérir un certain renom en médecine, d'élargir l'horizon de leurs connaissances en entreprenant un périple académique au lendemain de leur thèse.

$\mathrm{Si}$, avant de s'illustrer à la faculté de Strasbourg, Victor Stoeber pourra nous laisser des témoignages vécus sur le climat médical parisien au début du deuxième quart du $\mathrm{XIX}^{\mathrm{e}}$ siècle, il ne bénéficiait en rien de circonstances prédisposantes. Une heureuse fortune veut que ses impressions nous soient parvenues par le moyen d'une notice autobiographique assez peu connue ${ }^{1}$. Rédigés au soir de sa vie, le jour de Noël 1867, à l'intention de ses enfants, les souvenirs n'en conservent pas moins une vérité documentaire intéressante à plus d'un titre ${ }^{2}$.

Dernier de sept fils, Victor Stoeber est né le 16 février 1803. Quatre ans et demi plus tard, son père décède des suites d'un accident de voiture. Receveur général du Département, après avoir été emprisonné sous la Terreur, celui-ci avait été obligé de faire des avances considérables au gouvernement, voire des emprunts à des particuliers. «Mais un homme comme Napoléon I ${ }^{\text {er }}$ n'admettait pas ces raisons quand il lui fallait de l'argent.» Et plus tard, la Restauration ne se souciera guère de payer les dettes de l'Empire... Dans la famille, dont les ascendants possédaient le droit de bourgeoisie dans la cité depuis la Renaissance, la gêne s'installe. Aux restrictions s'ajouteront, pour le jeune élève du gymnase protestant, les rigueurs du premier blocus: «tous les animaux, chiens, chats, etc. avaient été mangés». Le retour des Bourbons amène la consternation, car les Stoeber ne comptaient que des «napoléonistes».

Et malgré les difficultés, Victor Stoeber se destine à la médecine. «Dès l'âge de dix ans, doit-il avouer, j'avais déclaré que je serais Docteur et Professeur.» Déterminant, dans ce choix, avait alors été «l'aspect du bienêtre» émanant de Thomas Lauth (1758-1826), auquel il était apparenté par sa grand'mère. Médecin traitant de la famille, le célèbre anatomiste arrivait en voiture traînée par deux chevaux blancs, vêtu d'une douillette en soie violette et l'hiver, enveloppé dans un manteau rouge garni de fourrure noire. 
Inscrit à la faculté depuis Pâques 1820, Victor Stoeber soutiendra sa thèse sur le delirium tremens - encore peu connu à ce moment - le 15 novembre $1824^{3}$. Conscient de l'insuffisance de l'instruction reçu, ambitionnant légitimement un avenir à la mesure de sa ténacité, il entreprend aussitôt un voyage académique, qui va durer trois années. Paris en est la première étape.

S'il ne nous est pas loisible, ici, de le suivre dans l'organisation matérielle de son séjour dont il rend un compte précis, il n'est pas inintéressant de retenir sa première installation dans la maison des éditeurs strasbourgeois Treuttel et Wurtz, située 17, rue de Lille.

Dépensant «le moins possible», il pourra améliorer ses ressources en rédigeant des analyses bibliographiques d'ouvrages allemands pour le Bulletin général et universel des annonces et des nouvelles scientifiques, dédié aux savants de tous les pays et à la librairie nationale et étrangère, publié sous la direction du baron de Férussac ${ }^{4}$, et édité chez ses logeurs. Il faut ajouter que la $3^{\mathrm{e}}$ section du périodique, connue sous le titre moins pompeux de Bulletin des sciences médicales comptait parmi ses collaborateurs toutes les sommités parisiennes, tels Andral, Béclard, Bourgelat, Cuvier, Flourens, Magendie, Pinel père et fils, Desgenettes, Laennec, Orfila, Lisfranc, Dubois fils, J.Cloquet, Sanson, etc. Stoeber les approcha, en même temps que les étrangers de passage, les voyageurs célèbres, les grands navigateurs, au cours des réunions hebdomadaires qui se tenaient chez Férussac.

Fréquentant avec régularité les séances de l'Académie des Sciences et de celle de Médecine, il a l'occasion de connaître les vénérables aînés, Portal, Lamarck, Chaussier, Jussieu.

Curieux de tout, il suit aussi les cours de chimie de Thénard et d'Orfila, ceux de physique de Gay-Lussac, ceux de zoologie de Blainville. Et aussi les leçons de littérature française de Villemain, d'histoire de Guizot et parfois il assiste aux cours de philosophie de Cousin. «Tous ces professeurs à cette époque, attiraient la foule, les uns par leur science, la plupart, en même temps, par l'élégance de leur débit.» Pour le jeune Alsacien, l'attrait devait assurément être très vif.

Son but, cependant, était la fréquentation des services cliniques. Le premier rendez-vous commence dès six heures à l'Hôtel-Dieu. Avec le talent exceptionnel qui était le sien, Mondor a reconstitué ces «matins de tous les jours» dominés par le prestige de Dupuytren ${ }^{5}$. Plus modestement, le portrait de celui qui restera encore pour Victor Stoeber, quarante années plus tard, «le modèle du professeur de clinique chirurgicale»n’en est pas moins saisissant d'objectivité: 
«Calme, impassible pendant les opérations, qu'il faisait avec une sûreté et une élégance extraordinaires, il exposait les faits lentement, et avec une clarté telle, que chaque mot se gravait dans la mémoire des élèves. D'une exactitude rigoureuse, il ne marchandait pas son temps, et bien des fois je l'ai vu, arrivé à 6 h., ne quitter l'hôpital qu'à 11 heures; il tirait alors de sa poche un petit pain qu'il mangeait, en se rendant chez lui près du Louvre. Pour suivre sa clinique, il fallait une carte qu'il délivrait lui-même. Assis à une petite table dans une salle de l'Hôtel-Dieu, il recevait les demandes. Les élèves lui présentaient leur inscription, les Docteurs leur diplôme; il donnait la carte aux élèves sans bouger, mais il saluait les docteurs. Lorsque je me présentai, je n'avais pas encore mon diplôme, mais on m'avait délivré à la faculté un certificat qui constatait que j'avais soutenu une thèse sur le delirium tremens. Je lui remis ce certificat; il me demanda alors si l'on connaissait à Strasbourg ses recherches sur le délire nerveux et si l'on se servait de son traitement par l'opium. Je ne m'étais pas encore, à cette époque, débarrassé d'une timidité extrême qui m'a longtemps poursuivi; je répondis cependant avec plus d'assurance que je ne m'en croyais capable, au milieu de cette foule d'étudiants, que l'on connaissait son traitement et qu'on l'employait quelquefois, mais pas toujours ${ }^{6}$. Là dessus, il me salua et me donna ma carte d'entrée.»

Sans doute, Dupuytren eût-il pu mettre l'auditeur débutant en confiance en lui rappelant la parfaite connaissance qu'il avait de la faculté de Strasbourg à la suite de sa mission de 1817 en qualité d'inspecteur général de l'université, qui aboutit à la création de la première chaire d'anatomie pathologique pour Jean-Frédéric Lobstein, le maître préféré de Stoeber. Trop mesuré, le temps lui manquait pour engager la confidence.

Très différente fut l'impression laissée par les démonstrations stéthoscopiques de Laennec. A la clinique du «petit homme grêle, qui avait l'air aussi malade que les individus couchés dans son service», il est révolté par l'«inhumanité» avec laquelle les Anglais se jettent sur les pauvres patients. Aussi préfère-t-il s'exercer dans les cliniques moins encombrées de Fouquier et de Lherminier ${ }^{7}$.

Plus sévère encore est le souvenir laissé par Broussais. Pendant quelque temps seulement, Stoeber suit sa visite au Val-de-Grâce. Ajoutons simplement qu'à ce moment le fougueux doctrinaire venait de publier son Cathéchisme de la médecine physiologique (1824).

«Curieux de voir si la pratique du célèbre réformateur était conforme à sa théorie», il fut «surpris de le voir assez souvent employer l'opium et même la quinine, remèdes incendiaires suivant la doctrine». Ne faisant pas encore partie de la faculté, Broussais est cependant «à l'apogée de sa gloire». Ayant quitté la rue du Foin, il venait d'inaugurer devant une «foule immense» ses cours libres dans le hangar de la rue des Grès. C'est là que Stoeber venait l'écouter après dîner. Il a gardé en mémoire l'image du despote «avec sa 
grosse tête entre les épaules, lançant les éclairs de ses petits yeux par dessus ses lunettes dorées et fulminer l'anathème contre ce qu'il appelait les ontologistes». Mais le narrateur sait aussi que «peu d'années après, il prêchait dans le désert, dans le grand amphithéâtre de l'école».

Avec régularité il va, en revanche, se rendre aux leçons cliniques de la Salpétrière. Rostan y combat le système de Broussais, «en soutenant toutefois les doctrines absolues de l'organicisme».

Portant déjà intérêt aux cliniques spéciales, parcimonieusement dispensées une ou deux fois par semaine en fin de matinée, il fréquente celles de Guersant à l'Hôpital des enfants rue de Sèvres, de Biett à Saint-Louis, d'Esquirol sur les maladies mentales à la Salpétrière, de Larrey au GrosCaillou. Son ami, le $\mathrm{D}^{\mathrm{r}}$ Wessely, futur médecin du prince d'Oldenburg, l'introduit chez Civiale et lui fournit «l'occasion de voir l'une des premières opérations de lithotricie».

Traversant la capitale au pas de course, il finit «par connaître [son] Paris comme [s'il n’avait] jamais vécu que là», pourra-t-il dire. Partageant les après-midi entre les cours de la Sorbonne et l'étude à son domicile, il assiste le soir aux leçons privées d'Andral sur l'anatomie pathologique.

Dans ces conditions, l'année scolaire avait passé rapidement. Mettant les vacances à profit, Stoeber visite Rouen, le Havre et Dieppe. Puis il entreprend avec deux amis strasbourgeois un véritable tour de France, le conduisant - le plus souvent à pied, plus rarement en diligence - d'Orléans à Nantes, de la Vendée à Bordeaux, puis dans le midi, pour revenir de Marseille à Lyon avant de rejoindre Paris. Il y demeurera encore jusqu'au mois de mai 1826.

Dès le début de son séjour à Paris Stoeber suivait les cours de crânioscopie de Gall (1758-1828). Un neveu strasbourgeois du célèbre phrénologue, ami de son frère, l'avait introduit dans la maison de son oncle. C'est ainsi que Gall, dont Stoeber rappelle la «très belle clientèle, surtout dans la colonie allemande, chez les ambassadeurs des états allemands et des souverains du Nord», lui propose de l'assister et de le remplacer auprès de ses malades. Bientôt septuagénaire, il est fatigué et cherche à diminuer ses occupations. La proposition est d'autant plus avantageuse que Stoeber peut prévoir qu'au bout de quelques années, il pourrait le remplacer complètement, «soit qu'il se retirât de la clientèle, soit qu'il vînt à mourir»...

Fort heureusement, il gardait sa fidélité à sa ville natale et avoue ne point aimer Paris. Aux séductions d'une «clientèle étendue et lucrative», il préfère poursuivre son instruction. L'Angleterre devait constituer une deuxième 
étape. Ayant étudié la langue anglaise tout seul à travers la traduction du «Vicaire de Wakefield», il bénéficie des leçons de prononciation d'un jeune confrère nommé Lilburn.

De fait, le voyage à Londres va décider de son orientation future. Il y retrouve en effet son ami Baum (1799-1883), rencontré à Paris - le futur titulaire de la chaire de chirurgie à Gœttingen - qui le présente à Wardrop et à Lawrence. Tous deux sont déjà spécialisés dans le traitement des maladies des yeux. Le premier exerce dans un hôpital privé de Santon Square, le second au dispensaire ophtalmologique de Moorfield.

Il peut alors établir un parallèle avec l'état où se trouvait l'ophtalmologie à Paris:

«Jusqu'alors, je n'avais aucune idée des maladies des yeux; notre professeur de pathologie externe nous avait fait une leçon sur l'ophtalmie, une sur la cataracte, et une sur l'amaurose. A la clinique, il n'entrait jamais de maladies oculaires, et en fait d'opérations, je n'avais vu avec ma lorgnette, du haut de l'amphithéâtre, que des opérations de cataracte et de fistules lacrymales pratiquées par Dupuytren et Roux. Je fus donc bien surpris de voir le nombre considérable d'affections oculaires qui tous les jours se présentaient à l'ophtalmic Infirmary Moorfields. A force d'en voir, je me familiarisai avec le diagnostic et le traitement de ces maladies, et je pris goût à cette partie de la science.»

Par la suite, il va fréquenter durant le semestre d'hiver 1827, un enseignement régulier de l'ophtalmologie sous la direction de Jüngken (1793-1875) à la Charité de Berlin, encore isolée au milieu des champs. A Vienne, il consacrera à nouveau la majeure partie de son temps à la fréquentation de la clinique ophtalmologique de Rosas (1791-1855) à l'Hôpital général et surtout à celle de Friedrich Jaeger (1784-1871), gendre de Beer, au Josephinum et à sa clinique privée. C'est auprès de lui qu'il va se lier intimement avec Jules Sichel (1802-1868), qui va bientôt fonder l'ophtalmologie parisienne à titre privé.

Stoeber est alors «persuadé [qu'il] en savait plus sur les maladies des yeux que [ses] futurs confrères de Strasbourg et même que l'immense majorité des médecins français. C'était malheureusement vrai. L'étude des maladies des yeux, qui avait tant fleuri au $18^{\mathrm{e}}$ siècle, y était depuis la révolution complètement négligée et la pratique en était abandonnée aux charlatans ou oculistes ambulants; au point qu'à Strasbourg, les chirurgiens ne faisaient plus d'opérations de cataracte, et que chaque année un Dr Forlenze, qui parcourait la France, arrivait et faisait à l'hôpital les opérations d'oculistique sur les pauvres qu'on y rassemblait. Un soi-disant officier de santé oculiste y arrivait encore pendant les dix ou quinze premières années de mon séjour et placardait les murs de ses affiches charlatenesques.» 
Le contrat, par lequel il s'était engagé à un âge habituellement réservé aux jeux, Victor Stoeber va le remplir pleinement après son retour à Strasbourg, en octobre 1827. Trois années durant, alors qu'il avait étudié quatre ans à la faculté, il s'était consacré à une formation complémentaire par un rythme de travail aujourd'hui confondant. Dès l'institution du premier concours d'agrégation, il est nommé en 1830, grâce à l'étendue de ses connaissances ${ }^{8}$. Au décès de Lobstein, en 1835, la chaire de clinique est mise au concours pour l'année suivante. Dans le but de mieux s'y préparer et de se «mettre bien au courant de tout ce qui se faisait à Paris», Stoeber s'y rend en été 1835. Il se lie d'une indéfectible amitié avec Louis, à la Pitié. Mais il y contracte aussi la rougeole et à son retour, une fièvre typhoïde se déclare... S'il n'est pas nommé à la chaire, malgré sa thèse d'une originalité et d'une actualité remarquables sur le rôle de l'anatomie pathologique ${ }^{9}$, il aura la satisfaction d'être élu («sans l'avoir jamais demandé», précise-t-il) correspondant national pour la division de médecine à l'Académie le 31 décembre 1836. Enfin, par un arrêté du 30 décembre 1845, ses ambitions sont satisfaites et il devient professeur de pathologie et de thérapeutique générales ${ }^{10}$.

A Paris, il avait naguère cherché les racines de l'expérience pour élargir son instruction. Proclamant que ce complément devait être obligatoire, Caffe le cite en exemple ${ }^{11}$. Sa perspicacité, cependant lui avait aussi fait découvrir les aspects négatifs de la médecine parisienne. Acquis à la nécessité d'un enseignement des spécialités, il se souviendra de la mentalité de «certains» médecins, et surtout de «certains» chirurgiens, se voulant «encyclopédistes [...] ce qui voudrait dire: connaître également bien toutes les parties de la médecine. C'est demander l'impossible.» D'ailleurs le public ne s'y est pas trompé. Et on a même vu des «chirurgiens célèbres, très-opposés aux spécialistes, s'adresser à Civiale et à Leroy d'Etiolles, lorsqu'ils ont été atteints de calculs de la vessie». ${ }^{12}$

Avec fierté, Stoeber pourra proclamer l'ouverture, par lui, de conférences théoriques, rapidement associées à des consultations sur les maladies des yeux, dès la fin du concours d'agrégation, en 1830, deux années avant Sichel. En 1834 fut publié son Manuel d'ophtalmologie ${ }^{13}$, alors le plus complet en France, et qui eut «les honneurs d'une contrefaçon à Bruxelles». Grâce au concours efficient de l'administration hospitalière, la mise à disposition d'un service particulier, dont Stoeber souligne le caractère indispensable, devient réalité. Ces mêmes conditions n'ayant pas été remplies à Paris, il avait assisté aux «résultats déplorables [...] dans les services de Dupuytren, de Boyer, de Roux». ${ }^{14}$ En 1853, la clinique ophtalmologique de Strasbourg devient 
officielle et figure au programme universitaire sous la direction de Stoeber. Comme le soulignera son ami Tourdes, «c'est cette situation de la médecine vis-à-vis d'une de ses branches importantes que Stoeber a complètement changée en Alsace et qu'il a contribué à modifier dans le reste de la France». ${ }^{15}$ Secondé par l'initiative du Doyen Coze, il assume également la clinique des maladies des enfants depuis le $1^{\text {er }}$ novembre 1837 jusqu'en 1845, puis, quoique avec moins d'enthousiasme, il réorganise celle des affections syphilitiques et cutanées.

Manifestement, une telle ouverture à l'organisation d'une médecine hospitalière nouvelle devait contribuer à la réputation de la faculté de Strasbourg sous le signe d'un particularisme constructif.

\section{Notes et bibliographie}

${ }^{1}$ Stoeber, Victor. A mes enfants (Notice biographique datée de Strasbourg, le 25 décembre 1867). 32 ff. dactylographiés. B.N. U. Als. cote M 41640. Le manuscrit original de ce texte que nous n'avons pas pu retrouver, est authentifié par des extraits cités par G. Tourdes dans l'éloge prononcé en 1871 (cf. infra).

2 Atteint depuis l'automne 1866 d'une affection des voies urinaires, Stoeber poursuivit ses activités, tout en ayant conscience de la gravité de son état. Indifférent au danger, il donnait encore ses soins aux malades dans les caves, dans les quartiers les plus exposés pendant le siège de la ville. Son gendre Ch. E. F. Monoyer, professeur agrégé à la faculté et son ami G. Tourdes se sont chargés de déclarer son décès, survenu le 5 juin 1871 en son domicile, 19 , rue de l'Ail (Arch. mun. de Strasb., Reg. des décès 1871/I, nº 1543).

${ }^{3}$ Stoeber, Victor. Dissertation sur le delirium tremens. F.-G.Levrault, Strasbourg 1824, in $4^{\circ},[$ IV] 37 p.

${ }^{4}$ Férussac était «officier supérieur au Corps royal d'Etat-Major», chevalier de Saint-Louis et de la Légion d'Honneur. La première livraison, pour 1824, comprenait 4 vol. in-8 ${ }^{\circ}$. Le périodique prit ensuite le nom de «Bulletin universel des sciences et de l'industrie», 1824-1835, 166 vol. in-8 ${ }^{\circ}$. Il était divisé en 8 sections, dont la $3^{\mathbf{e}}$ était consacrée aux sciences médicales (27 vol.), dirigée par le $\mathrm{D}^{\mathrm{r}}$ de Fermon. Stoeber poursuivra sa collaboration jusqu'en 1862, qui comprendra 28 articles bibliographiques depuis 1826 (cf. E. Hatin, Bibliographie historique et critique de la presse périodique française. Didot, Paris 1866, p. 575).

${ }^{5}$ Mondor, Henri. Dupuytren. $2^{e}$ éd. Gallimard, Paris 1945. 312 p. Chap. X, p. 137-153.

${ }^{6}$ En fait, il avait écrit, à ce propos dans sa dissertation (p.31): «Ce n'est que dans les cas déjà spécifiés par Sydenham, Boerhaave, Van-Swieten, et dans les cas désespérés, que j'employais l'opium.»

7 Vetter, Th., Jean-Frédéric Lobstein le Jeune et l'introduction de la stéthoscopie à la faculté de Strasbourg. Rev. du Palais de la Découverte. No spécial 22 août 1981, p. 138-151.

${ }^{8}$ Stoeber, Victor. Diss. De hydrope ventriculorum cerebri. Competitio ad agregationem. Typ. Videae Silbermann, Argentorati s.d. (1829), in- $4^{\circ}, 34$ p. 
${ }^{9}$ Stoeber, Victor. Appréciation des progrès que l'anatomie pathologique a fait faire à la thérapeutique. Thèse présentée et soutenue... le 12 février $1836 \ldots$ pour le concours de la chaire de clinique interne. F.-G. Levrault, Strasbourg 1836, in- $4^{\circ}, 49$ p.

${ }^{10}$ Stoeber, Victor. De l'influence que l'analyse chimique et la micrographie ont exercée sur la pathologie et la thérapeutique. Thèse de concours présentée et soutenue devant le jury... le 9 avril 1845. Impr. G. Silbermann, Strasbourg 1845, in- $4^{\circ}, 99$ p.

${ }^{11}$ Caffe, Nécrologie de Victor Stoeber. Journ. des connaissances méd. et pharm. XXXIX, 4 (1871) p.64.

${ }^{12}$ Stoeber, Victor, De l'enseignement des maladies des yeux et de l'exercice de cette spécialité (Discours prononcé à l'ouverture de la Clinique ophtalmologique de la faculté de médecine de Strasbourg, le 6 avril 1869), Gaz. méd. de Strasb. 31, $3^{\mathrm{e}}$ série, $\mathrm{n}^{\circ} 18$ (1872) p. 209-213.

${ }^{13}$ Stoeber, Victor. Manuel pratique d'Opthalmologie ou Traité des maladies des yeux. Ed. F.-G. Levrault, Paris 1834, in-8 ${ }^{\circ}$, XIV, 406 p., 3 pl.

${ }^{14}$ De l'enseignement des maladies dex yeux. Op.cit. p. 211.

15 Tourdes, Gabriel. Notice biographique sur Victor Stoeber. Gaz. méd. de Strasbourg, XXXI, 7-9, 1871, p.82-87, 93-98, 104-107, p.93. Cette notice est suivie d'une bibliographie raisonnée des travaux de Stoeber. Elle a servi de modèle à l'article biographique rédigé par Louis Hahn pour le Dictionnaire des sciences médicales de Dechambre, $3^{\mathrm{e}}$ série, T. 12 (1883) p. 135-138.

Le texte de Tourdes parut également, sans les éléments bibliographiques, dans la Gazette des Hôpitaux civils et militaires - La Lancette Française, XLIV, 1871: ${ }^{\circ}$ 137, p. 545-546; $\mathrm{n}^{0} 139$, p.553-555; $\mathrm{n}^{0} 141$, p. 561-563; n $\mathrm{n}^{0} 142$, p. 565-566; n $\mathrm{n}^{\circ} 147$, p.585-586; $\mathrm{n}^{\circ} 148$, p. 589-590 de même que dans les Mémoires de la Soc. de méd. de Strasbourg, T.IX (1872) p. $1-39$.

Dans le discours prononcé lors des obsèques par le Doyen Stoltz au nom de la faculté (Gaz. méd. de Strasb., XXI, 4, 1871, et Gaz. des Hôpitaux civ. et mil., XLIV, 25, 1872, p. 97-98, et Rev. de thérap. méd. chir. 38, 24, 1871, p.670-672) l'orateur ne fait pas mention du séjour parisien de Stoeber, de même que Boeckel dans l'éloge prononcé au nom de la Soc. de méd. (Gaz. méd. de Strasb., XXXI, 4, 1871, p.39-40).

\section{Summary}

During a complementary study trip of three years started at the end of 1824, Victor Stoeber got familiarized during one year and half with the Paris medical life. The special clinics wo which he devoted a particular interest were still parcimoniously organized. Attracted by ophtalmology in England he then perfected himself in Berlin and Vienna. After his return in Strasbourg he gave theoretical lessons associated to consultations on eye diseases as soon as 1830. When elected in the chair of general pathology and therapeutics he had ophtalmology included in the official program of the Faculty in 1853. His souvenirs left in his autobiography allow to place the "strasburgian particularism" in relation with the mentality of the parisian celebrities which were during a long time opposed to specialization.

Dr. med. Théodor Vetter, 12, rue des Orfèvres, F-67070 Strasbourg 